\title{
Entity Linking across Vision and Language
}

\author{
Aparna Nurani Venkitasubramanian . Tinne \\ Tuytelaars • Marie-Francine Moens
}

Received: date / Accepted: date

\begin{abstract}
We propose a novel weakly supervised framework that jointly tackles entity analysis tasks in vision and language. Given a video with subtitles, we jointly address the questions: a) What do the textual entity mentions refer to? and b) What/ who are in the video key frames? We use a Markov Random Field (MRF) to encode the dependencies within and across the two modalities. This MRF model incorporates beliefs using independent methods for the textual and visual entities. These beliefs are propagated across the modalities to jointly derive the entity labels. We apply the framework to a challenging dataset of wildlife documentaries with subtitles and show that this integrated modelling yields significantly better performance over text-based and vision-based approaches. We show that textual mentions that cannot be resolved using text-only methods are resolved correctly using our method. The approaches described here bring us closer to automated multimedia indexing.
\end{abstract}

Keywords Entity Linking · Animal Labeling · Multimedia Indexing · LanguageVision Alignment

\section{Introduction}

It is estimated ${ }^{1}$ that it would take an individual more than 5,000,000 years to watch the amount of video that will cross global Internet Protocol (IP) networks each month

Aparna Nurani Venkitasubramanian

KU Leuven, Computer Science Department, Celestijnenlaan 200A, B-3001 Leuven, Belgium

E-mail: aparna.venkit@gmail.com

Tinne Tuytelaars

KU Leuven, ESAT-PSI, Kasteelpark Arenberg 10, B-3001 Leuven, Belgium

E-mail: tinne.tuytelaars@kuleuven.be

Marie-Francine Moens

KU Leuven, Computer Science Department, Celestijnenlaan 200A, B-3001 Leuven, Belgium

E-mail: sien.moens@kuleuven.be

1 http://www.cisco.com/c/dam/en/us/solutions/collateral/service-provider/visual-networking-indexvni/complete-white-paper-c11-481360.pdf 


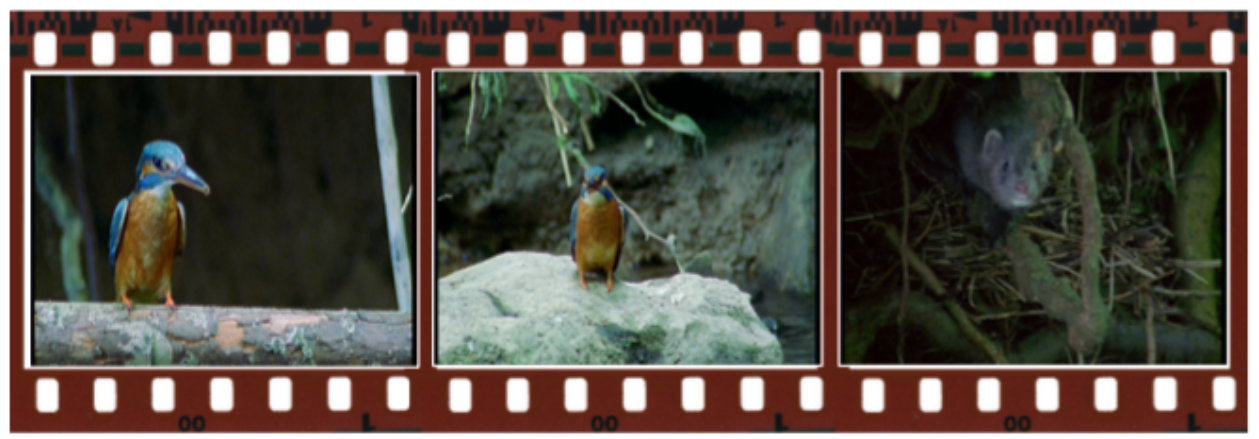

[...] this daybreak finds the kingfisher still digging. She ${ }_{1}$ must be desperate. [...] A mink. I thought it was an otter when it burst out from the bank. One kingfisher had dived to safety, but which one? It was impossible to tell. The mink had been waiting in ambush, hidden, even from me, almost certainly attracted by the kingfishers' frantic whistling. She ${ }_{2}$ stashed the first bird and returned, sure that there was another. But one kingfisher got lucky. She 3 spotted me.

Fig. 1 An example of a subtitle excerpt together with the associated frames

in 2020. Therefore, it is imperative that we have tools that will enable us to search and find not only relevant videos in a corpus, but also relevant snippets within a video. Towards this goal of making videos 'searchable', we consider a wildlife documentary with subtitles and address two problems that typically occur in such videos: a) Mapping the mentions in the subtitle to the correct animal name (Entity linking); and b) Identifying animals in the video key frames (Animal labeling).

These seemingly unrelated problems are quite closely coupled in reality. Particularly in videos, language and vision are complementary to each other and it is essential to look at them in unison. Vision tasks often benefit from the associated text [42] while Natural Language Processing (NLP) tasks benefit from the vision. As an example, consider Figure 1. Here she ${ }_{1}$ refers to the kingfisher and she ${ }_{2}$ refers to the mink. While there is no ambiguity in these two cases, resolving she ${ }_{3}$ is not straight-forward. This piece of text might suggest that $\mathrm{she}_{3}$ refers to the kingfisher, but in reality, it refers to the mink. The use of the associated frames makes this clear. Especially in multi-modal settings such as ours, the visual component is crucial for the correct resolution of the textual mentions. In this work, we tackle the problem of entity linking and animal labeling in text and vision jointly.

In a weakly supervised setting, this problem presents a host of challenges for vision, text and the association of text and vision. On the vision side, we deal with a scenario where there are no visual demarcators to indicate the location of an animal. In fact, it is not even known if there are animals at all in a certain key frame. Additionally, since we are dealing with animals shot in their natural habitat, there are challenges due to self-occlusion, camouflage, illumination etc. On the textual side, while we have tools $[9,24]$ to detect entity mentions in the text, not all of them are pertinent to animals. Even when the mentions refer to animals, they are often so ambiguous (e.g. 'targets for the crocodile' and 'the predators') that it is impossible to resolve them correctly without a holistic understanding of the context. As far as the linking of text and vision is concerned, the absence of bounding boxes in the visual 
data coupled with the presence of ambiguous mentions in text makes it harder to reliably tie together the entities in vision and language, that is, there are no ready examples to show the association in a limited, diverse dataset.

In order to address the animal labeling task in vision and the entity linking task in language, we build a Markov Random Field (MRF) using the textual and visual entities. The MRF models the dependencies that exist in language (among the various mentions), in vision (among the frames) and across language and vision (depicting connections between a mention and an animal shown). For the textual entities, we use the state-of-the-art coreference resolution system of Durrett and Klein [9] and for the visual entities, we use the model of Venkitasubramanian et al. [42] which predicts animal labels in vision on a frame-by-frame basis. Using these as starting points, we apply belief propagation to draw inferences on text and vision jointly. Here, we use a structured predictor that leverages the continuity aspect inherent in videos. Building on the approach of Venkitasubramanian et al. [42], we not only improve the recognition in vision using text, but also address the problem of entity linking in text using vision.

The key contributions of this paper are as follows:

1. We propose a novel probabilistic framework to jointly map the entities in vision and language, by capturing interdependencies within and across the modalities.

2. We propose a method to filter mentions and retain only those relevant for the context. In this work, we focus on the detection of mentions pertinent to animals.

The rest of this paper is organized as follows: Section 2 discusses related work. Section 3 defines the problem. Section 4 describes our framework and Section 5 describes the detection of relevant mentions. In Section 6, we provide the implementation details. Section 7 describes the experiments and results. Finally, Section 8 provides the conclusions and future work.

\section{Related Work}

To the authors knowledge, this is the first work that addresses the problem of entity linking across language and vision. Our task is at the confluence of a few other tasks: 1) Entity analysis tasks in text, 2) Animal labeling in vision, 3) Aligning text and vision and 4) Cross-modal coreference resolution. In what follows, we describe the related work in each of these domains.

\subsection{Entity Analysis Tasks in Text}

The entity analysis stack in text comprises three tasks: named entity recognition, coreference resolution and entity linking.

While early work [6] formulates Named Entity Recognition (NER) as recognizing 'proper names' in general, the scope has since been widened to include certain 'natural kind terms like biological species and substances' [29]. Alfonseca and Manandhar [2] define NER as the task of finding and classifying objects that are of interest 
to us. The need for precise NER tools has lead to the development of several domainspecific approaches. For example, in the biomedical domain, several methods have been proposed to recognize gene or protein names, diseases, drugs etc. [25]. In our work, we focus on animals and identify the referents using a list of animal names from WordNet [28]. Additionally, we propose an approach to detect other mentions that are pertinent to animals using one of the most salient features used in NLP tasks, that is especially relevant to sentient beings - animacy. Furthermore, we propose a more generic method for detecting relevant mentions, that can deal with a wider class of objects that is not restricted to animals.

Coreference resolution is the task of finding all expressions that refer to the same entity in a text. The state-of-the-art probabilistic coreference resolution system is the model of Durrett and Klein [9]. The system is basically a Conditional Random Field (CRF) that takes in a set of 'surface-level' or general purpose features together with a set of more sophisticated 'semantic' features (such as hypernymy, synonymy etc.). The state-of-the-art deterministic coreference resolver is that of Lee et al. [24]. This system relies on a set of rules or sieves applied one at a time in the order of decreasing precision.

The entity linking task focuses on mapping an entity to an entry in a knowledgebase. For an overview of various approaches to entity linking, we refer to [41]. The entity linking task is usually preceded by a Named Entity Recognition (NER) task. The classical entity linking task is already quite challenging due to name variations and entity ambiguity. In our setting, these challenges are far more pronounced. Resolving the mention 'targets for the crocodiles' to 'zebra' is far more difficult compared to resolving, for example, 'Cornell' to 'Cornell university' - the search space is much wider and the desirable outcome ('zebra') is not apparent from the words in the mention.

Yet another interesting work is that of Durrett and Klein [10], where all the three tasks namely named entity recognition, coreference resolution and entity linking are tackled jointly. All the above approaches only apply to text. In contrast, we develop a method that exploits both textual and visual modalities to perform entity linking.

\subsection{Animal Labeling in Vision}

Animals are among the most difficult objects to recognize in images and videos, mainly due to their deformable bodies that often self occlude and the large variation they pose in appearance and depiction $[4,1]$. Further, in the natural habitat, there are challenges due to camouflage and occlusion by flora. Although identifying animals is a well-studied topic $([4,1,38,35])$, recent works such as those of Hariharan and Girshick [17] and Gomez et al. [14] advance us further and provide better insight into the problem. However, these methods are not applicable in our setting since they require extensive training data.

Apart from these works that focus specifically on animals, there is a large literature on generic object detection. These methods are often evaluated on the Pascal VOC challenge dataset [12] which includes classes of animals such as cats, dogs, cows and horses, among other things. There are also datasets that focus on animals 
such as Caltech UCSD Birds [44] and Stanford Dogs [22]. Additionally, the FishClef and BirdClef challenges which are part of LifeClef [19] provide an arena for identification of species of fish and birds respectively. Most of these datasets are, however, object-centered and in that sense easier than the 'in-the-wild' setting we are dealing with. In this work, we propose a rather generic framework using the features of vgg-net [5], which are activations of a convolutional neural network.

The problem of aligning animals from videos with their mentions in subtitles has been studied in [11] and [42]. The former approach relies on hand-annotated bounding boxes to localize the animals in a frame and uses an Expectation-Maximization (EM) algorithm to map the bounding boxes to the correct animal names. The latter learns classifiers from ImageNet [8] and adapts them to the target dataset using an EM algorithm. Our approach is different from these in a couple of ways. Firstly, both the approaches target only the vision side, while we address the problem on the language side as well. Secondly, even on the vision side, the approaches of [11] and [42] were applied only on a frame-by-frame basis, whereas our approach uses structured prediction that leverages the continuity of frames.

\subsection{Combining Text and Vision}

Recently, there has also been some work on alignment across modalities for recognizing people $[32,31,16]$. These approaches rely on the use of a face-detector. While there are face detectors available with reasonable accuracy, there are no such detectors that allow localizing animals. As noted by Venkitasubramanian et al. [42], the absence of the bounding boxes complicates the problem in many ways. Further, these approaches only use the names of people on the textual side, while we address a much broader problem of mapping any phrase that indicates an animal (e.g. the neighbor) to the right animal.

There has also been considerable interest in sentence/caption generation from images as well as natural language based object detection [20,13,15,21]. These approaches are not directly applicable to our setting. First, we have too few data to train similar models. Second, in our context, the subtitles and the visuals are not parallel, but complementary. For example, often a few animals are mentioned in the text, while the connected frame only shows one of them. The connection between the vision and the text is therefore much weaker.

\subsection{Cross-modal Coreference Resolution}

Ramanathan et al. [36] address the problem of coreference resolution in a multimodal setting involving people in video sequences together with their names in the text. While this approach also handles ambiguous mentions such as the man, the engineer etc. on the textual side, on the visual side, it is used in a much cleaner setting with faces in bounding boxes.

Another work that addresses coreference resolution in text and vision is that of Chen et al. [23] where images of furniture (represented as 3D cuboids) are mapped 


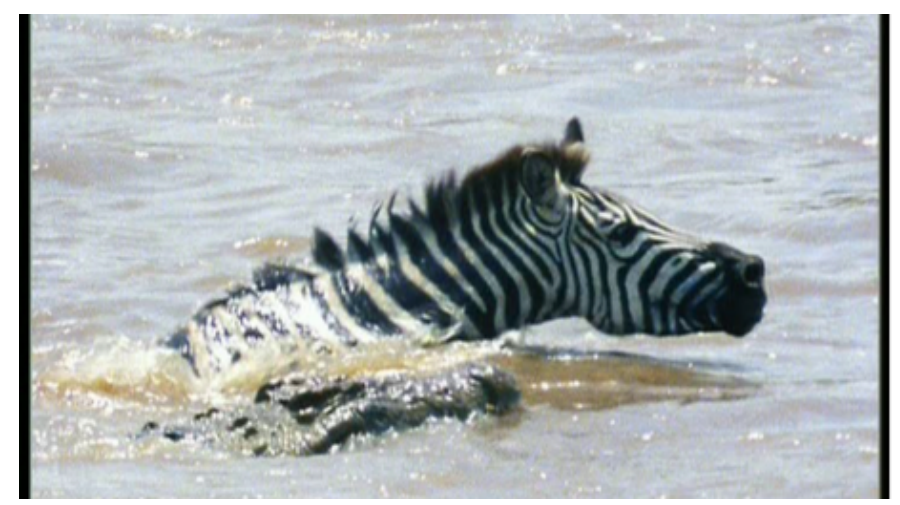

Crocodiles are everywhere. The migration is the bonanza they've been waiting for. But the zebra are surprisingly well-armed. Even in water, a zebra's kick is more than a crocodile can endure. As each family makes it safely to the other side, its members reassemble in a frenzy of greeting. But there are still plenty of targets for the crocodiles, who begin to step up their onslaught. Some foals $[\ldots]$

$\begin{aligned} \text { they } & \rightarrow \text { crocodile } \\ \text { each family } & \rightarrow \text { zebra } \\ \text { its members } & \rightarrow \text { zebra } \\ \text { targets for the crocodiles } & \rightarrow \text { zebra } \\ \text { their } & \rightarrow \text { crocodile } \\ \text { foals } & \rightarrow \text { zebra }\end{aligned}$

Fig. 2 An example of the entity linking task in text. The unique names identified for this caption are crocodile and zebra (underlined), the mentions to be resolved are in bold.

with natural language descriptions of the room. Our problem is much more complex in several ways. Firstly, on the vision side, we are dealing with animals which come in various shapes and flexible bodies instead of 3D cuboids. Second, on the textual side, we deal with more complex expressions such as the caravan of predators which do not explicitly state what they are referring to. Finally, the subtitles in our context are not intended to describe the visual appearances, but to augment them with extra information.

\section{Task Definition}

The input to our system consists of a wildlife documentary with subtitles. On the textual side, we have a set of sentences in the subtitles. These sentences contain mentions $\mathbf{M}=\left\{m_{1}, m_{2} \ldots m_{r}\right\}$. Of these mentions, we are only interested in those pertinent to animals ${ }^{2}$. These mentions include the nominal mentions and the pronominal ones. From the nominal mentions, we can derive the set of unique animal names such as penguin, lion etc. denoted by $\mathbf{N}=\left\{n_{1}, n_{2} \ldots n_{p}\right\}$. We do this by comparing each mention against a list of animal names derived from WordNet [28] as in [42].

\footnotetext{
2 We experiment with both gold mentions and those detected automatically using Section 5.
} 
On the visual side, we have key frames $\mathbf{F}=\left\{f_{1}, f_{2} \ldots f_{q}\right\}$ which may or may not contain animals. Using the setup of [42], every frame is linked to five subtitles to the left and right of the frame. All mentions in this range of subtitles are also associated with the frame. Thus, we have a set $\mathbf{P}$ of mention-frame pairs $\left\langle m_{i}, f_{j}\right\rangle$. Further, the unique names in this set of subtitles are also associated with the frame. Thus, with each frame $f_{j}$, we have a set of associated animal names $\mathbb{N}_{j}$.

Our objective is to jointly map the mentions $\mathbf{M}$ and frames $\mathbf{F}$ to the correct names $\mathbf{N}$. This is described by the two tasks below:

1. Animal labeling on the vision side $(\mathbf{F} \rightarrow \mathbf{N})$ : The task is to identify what animals are in the frame. For each name $n_{l} \in \mathbb{N}_{j}$ corresponding to frame $f_{j}$, we have a binary variable $y_{l}$ indicating presence or absence of animal $n_{l}$. The animal labeling task aims at identifying the most likely value of $y_{l}$ corresponding to name $n_{l}$ for frame $f_{j}$.

2. Entity linking on the text side $(\mathbf{M} \rightarrow \mathbf{N})$ : Entity linking tries to associate each mention with a knowledge base entry [26]. In our case, this 'knowledge base' is the list of animal names derived from WordNet [28] as stated earlier. For each mention $m_{i} \in \mathbf{M}$, we have a set of possible names, which are the same as those corresponding to the frames associated with $m_{i}$. That is, for every mention $m_{i}$, we have a set of associated frames $\mathbb{F}_{i}$ derived from the set $\mathbf{P}$ of mention-frame pairs. Each frame $f_{e} \in \mathbb{F}_{i}$ has a set of associated animal names $\mathbb{N}_{e}$. Then, the names associated with the mention $m_{i}$ are $\mathbb{N}_{i}=\bigcup_{e} \mathbb{N}_{e}$

The entity linking task aims at identifying the most likely name $n_{k}$ from animal names $\mathbb{N}_{i}$ corresponding to mention $m_{i}$. Figure 2 shows an example of this task. Note that this includes mapping mentions such as targets and victim to the right animal, but we do not make the distinction between the different members of the same species. For example, 'the zebra that swam across the river' and 'the one that watched him' will both refer to the animal Zebra. This is what makes our task different from a classical coreference resolution task.

\section{Our Approach}

We use a probabilistic graphical model, specifically a Markov Random Field (MRF), to denote the relationships between the frames and mentions over the entire video. We have two kinds of nodes:

- Visual nodes V: A frame may have several animals or none. To denote this, we use one node for every frame-animal name combination. This is a binary node that indicates the presence or absence of the animal in the frame.

$\mathbf{V}=\left\{v=<f_{j}, n_{k}>\mid n_{k} \in \mathbb{N}_{j}\right.$, the set of candidate names associated with $\left.f_{j}\right\}$

- Textual nodes T: As with the frames, we have one node for every mention-animal name pair to indicate whether a mention maps to a name. We consider all the mentions that need to be resolved.

$\mathbf{T}=\left\{t=<m_{i}, n_{l}>\mid n_{l}\right.$ is associated with frame $f_{e} \in \mathbb{F}_{i}$ corresponding to $\left.m_{i}\right\}$

Now, we build a bipartite graph $G=<\mathbf{V}, \mathbf{T}, E>$, with edges $E$ across the visual and textual nodes, $\mathbf{V}$ and $\mathbf{T}$ respectively. The edges are built between any 


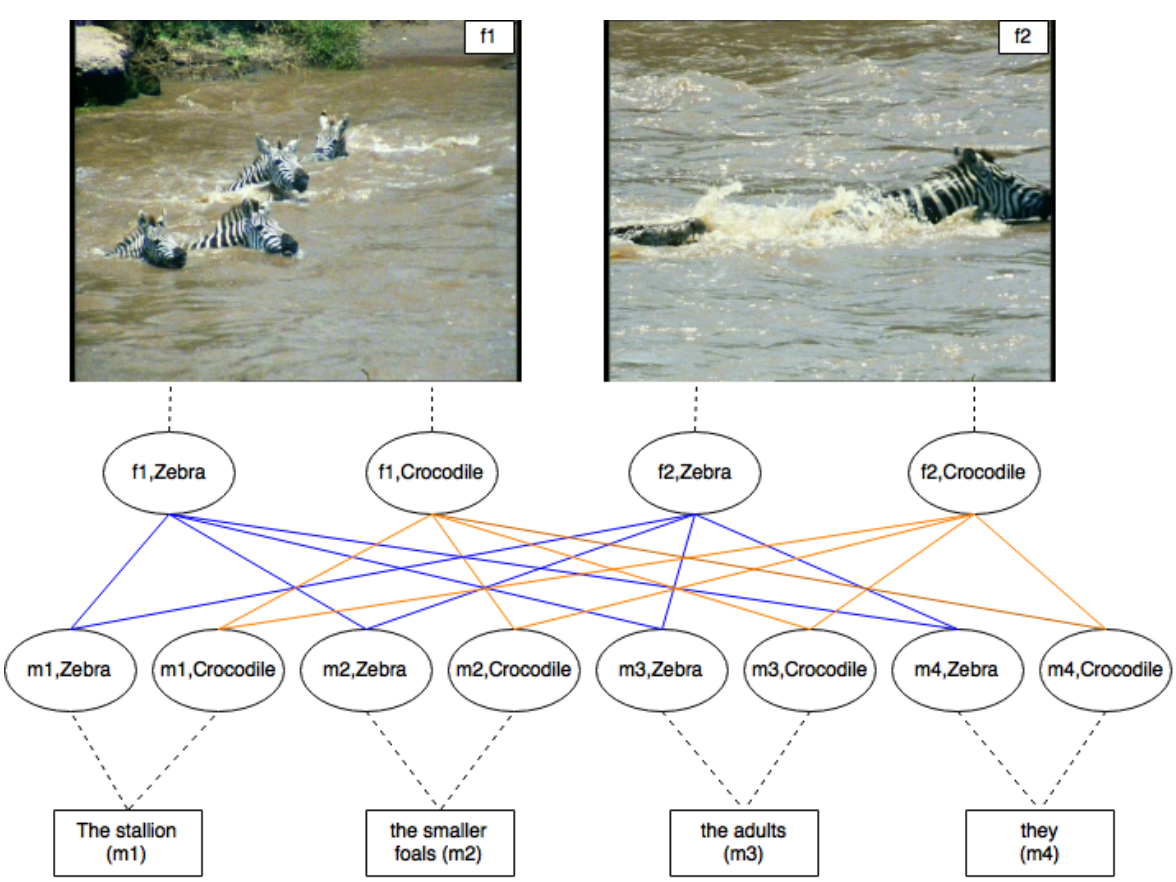

The stallion's responsibility is great, for the powerful current is more than strong enough to wash the smaller foals downriver. But the adults know that navigating the undertow is only part of the battle. There are other more sinister dangers. Crocodiles are everywhere. The migration is the bonanza they've been waiting for.

Fig. 3 An example of a part of the graphical model built using two frames (first row) and the corresponding subtitles (last row). In the subtitles (last row), the mentions to be resolved are in bold and the unique names are underlined. The ovals denote the nodes in vision (second row) and text (third row). The blue edges denote the links among the zebra nodes, while the orange edges denote the links among the crocodile nodes. The mentions to be resolved (The stallion, the smaller foals etc.) are shown in the rectangles.

pair of nodes $v \in \mathbf{V}$ and $t \in \mathbf{T}$ iff frame $f_{j}$ is associated with mention $m_{i}$ (that is, $\left.<m_{i}, f_{j}>\in \mathbf{P}\right)$ and $n_{l}=n_{k}$. Figure 3 shows an example of the graphical model for a snippet from our video. This is an episode on zebra and crocodiles. Note that this graph has two connected components (blue and orange), one for each animal name. Also note that the graph has cycles (loops).

We use a Markov Random Field to infer the values of the hidden labels. For this global inference over the video, we experiment with two different paradigms: 1) Message-passing and 2) Particle-based or sampling methods. For the message passing, we use the sum-product or Loopy Belief Propagation (LBP) algorithm. Figure 4 illustrates the message passing using the cluster graph for one connected component (Zebra) of Figure 3. Note that every edge influences one textual and one visual node each and messages are passed from one cluster to another through the textual or visual nodes. The use of the bipartite graph ensures that beliefs are propagated among the vision nodes, through the associated textual nodes and vice versa, allowing the two modalities to influence each other. Pearl [30] showed that the belief propagation algo- 


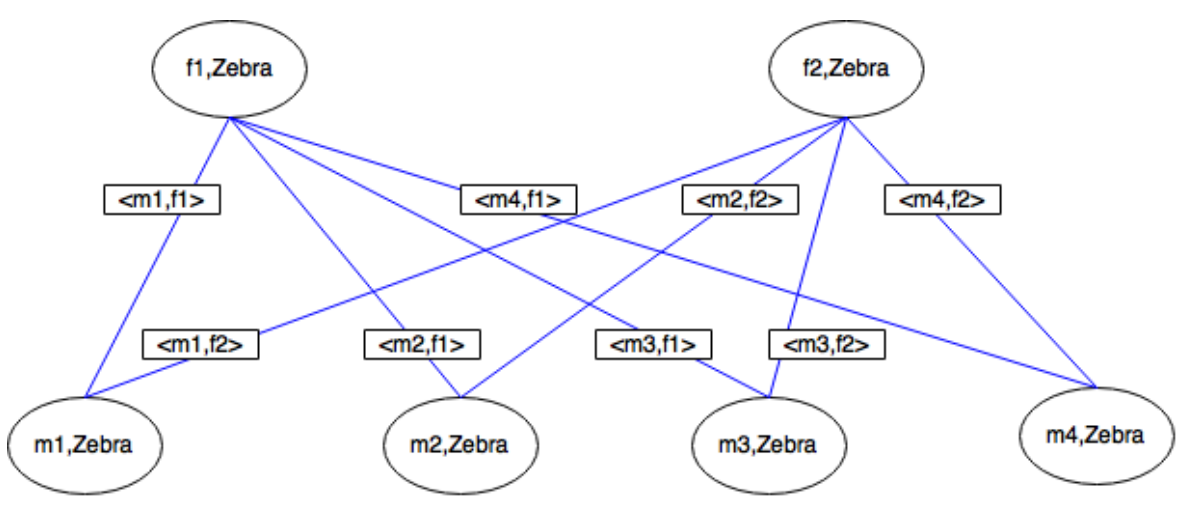

Fig. 4 An example of the cluster graph for one connected component (Zebra) of Figure 3. The ovals denote the nodes in vision and text (first and last rows respectively). The blue edges denote the links among the zebra nodes.

rithm is exact if the graph is a tree and approximate when the graph contains cycles. Since our graph has cycles, the inference is approximate in our case. LBP has been used successfully both in computer vision [7] and natural language processing [37]. We also experiment with Viterbi (VIT) Approximation (or max-product algorithm) that is quite similar to LBP but works in the log space.

Yet another approach to inferencing is through sampling. We experiment with Gibbs sampling, in addition to LBP and VIT. In Gibbs sampling, we randomly sample instances from the distribution and use those as a sparse representation, which are used to re-estimate the probabilities iteratively, thus preventing the algorithm from getting stuck in a local maximum.

The potential functions $\psi_{\text {text }}, \psi_{\text {vision }}$ and $\psi_{\text {text_vision }}$ are initialized as follows:

- For each textual node $t=<m_{i}, n_{l}>\in \mathbf{T}$, the node potential $\psi_{\text {text }}\left(m_{i}, n_{l}\right)$ is obtained using the state-of-the-art probabilistic coreference resolver of Durrett and Klein [9].

Their system estimates the probability that a certain mention is the back-pointer of some other mention, thereby generating a back-pointer probability matrix which will be used for our node potentials. We mirror the back-pointer probability matrix along the leading diagonal in order to have a symmetric matrix. Then, to obtain the node potential $\psi_{\text {text }}\left(m_{i}, n_{l}\right)$ corresponding to mention $m_{i}$ and animal name $n_{l}$, we look at all the back-pointer probabilities associated with mention $m_{i}$, and filter them by those containing the name $n_{l}$. For example, suppose the mention to be resolved $m_{i}$ is 'targets for the crocodiles', and we are interested in $n_{l}=$ Zebra. Then, we look at the row in the back-pointer probability matrix corresponding to this mention and all the columns where there is a 'head_match' with the name $n_{l}$. That is, we find all the columns where the head word is 'Zebra'. Since there could be multiple mentions that contain Zebra as the head word, we choose the maximum ${ }^{3}$ of these probabilities as the node potential

\footnotetext{
3 We use maximum of the probabilities instead of mean or minimum because the most influential candidates are those that are closer to the said mention and have a high pair-wise score in the back-pointer probability matrix.
} 
$\psi_{\text {text }}($ targets for the crocodiles,Zebra $)$.

$$
\begin{array}{r}
\psi_{\text {text }}\left(m_{i}, n_{l}\right)=\max _{x}\left\{p\left(m_{i} \rightarrow m_{x}\right) \mid\right. \\
\text { head_match } \left.\left(m_{x}, n_{l}\right)\right\}
\end{array}
$$

where $p\left(m_{i} \rightarrow m_{x}\right)$ denotes the back-pointer probability from mention $m_{i}$ to mention $m_{x}$.

- For each visual node $v=<f_{j}, n_{k}>\in \mathbf{V}$, the node potential $\psi_{\text {vision }}\left(f_{j}, n_{k}\right)$ is defined as the strength of the connection between the frame $f_{j}$ and name $n_{k}$. This is obtained from the probability $p\left(n_{k} \mid f_{j}\right)$ estimated using the model of [42].

- The edge potentials $\psi_{\text {text_vision }}\left(m_{i}, f_{j}\right)$ for the edges connecting the nodes are shared across the entire network and are obtained using a small validation set.

Using the above, the joint probability which will be maximized during the training of the network is given by

$$
P(\mathbf{V}, \mathbf{T})=\frac{1}{Z} \prod_{\substack{<m_{i}, f_{j}>\in \mathbf{P} \\<m_{i}, n_{l}>\in \mathbf{T} \\<f_{j}, n_{l}>\in \mathbf{V}}} \psi_{\text {text }}\left(m_{i}, n_{l}\right) * \psi_{\text {vision }}\left(f_{j}, n_{l}\right) * \psi_{\text {text_vision }}\left(m_{i}, f_{j}\right)
$$

where $Z$ is the normalization constant.

Finally, to obtain the entity labels on the vision side, for each node $v=<f_{j}, n_{k}>\epsilon$ $\mathbf{V}$ we assign the label $n_{k}$ to frame $f_{j}$ if the node potential $\psi_{\text {vision }}\left(f_{j}, n_{l}\right)>0.5$. This allows us to identify frames without any animal or with multiple animals of different species. On the textual side, since a mention typically refers to a single entity ${ }^{4}$, we look at all the node potentials $\psi_{\text {text }}\left(m_{i}, n_{l}\right)$, corresponding to mention $m_{i}$ and choose the name $n_{l}$ that has the highest node potential.

\section{Detecting Relevant Mentions}

The text contains several mentions that refer to animals. These include nominal mentions such as 'penguin', 'the male', 'the mother', 'victims', 'cubs' etc. and pronominal mentions such as 'she', 'he', 'they' etc. To detect the mentions pertinent to animals, we experimented with two approaches. The first relies on the 'animacy' feature of a state-of-the-art deterministic coreference resolver, while the second uses a large database of hypernym relations extracted from the Web. These approaches are explained in detail below.

\footnotetext{
4 It is possible that one mention actually refers to multiple animals. For example, 'The zebra and giraffe peacefully co-exist in Savannah. They are both very valuable to the wildlife... '. Here, the mention they refers to zebra and giraffe. However, we do not see such cases in our dataset, and ignore this case for simplicity.
} 


\subsection{Using the 'Animacy' Feature}

The problem of detecting mentions that are relevant for animals largely boils down to finding whether a mention is animate or not. To find whether a mention is animate, we turn to a state-of-the-art deterministic coreference resolution system that uses 'animacy' as a feature. The coreference resolution system of Lee et al. [24] uses a set of sieves or rules, one at a time, ordered from highest to lowest precision ${ }^{5}$. One of these sieves relies on matching attributes such as animacy, gender and number of a mention with its antecedent(s). The 'animacy' feature which was built into the system turned out to be the second most important feature (next to number) for pronoun resolution. Their system sets one of the three values: ANIMATE, INANIMATE or UNKNOWN for the 'animacy' attribute. To detect relevant mentions, we first matched the mentions predicted by the probabilistic coreference system Durrett and Klein [9] with those predicted by the deterministic one of Lee et al. [24]. Then, we concluded that a mention corresponds to an animal if the mention is ANIMATE according to the system of Lee et al. [24] or if the head word contains an animal name. The reason we included the check for the head word is that we observed that several animal names such as crocodiles, bear, dolphins etc. were classified as INANIMATE. We also excluded first (such as I, me, myself, mine) and second person pronouns (such as you, your, yours) since they typically refer to the presenter and the viewer respectively. Since we are interested in animals in this work, the animacy feature is well-suited to this problem. However, in order to apply this work to other contexts involving inanimate objects, we propose an alternative approach.

\subsection{Using a Hypernym Database}

The second approach for detecting relevant mentions uses the WebIsADb database [40] containing more than 400 million hypernymy relations extracted from the CommonCrawl web corpus. This framework allows us to query by two attributes: class and instance, both of which have the sub-attributes: prefix, lemma and suffix. The output of the system is the frequency of that hypernym pair. For example, by specifying that the lemma of the class name is 'animal' and the lemma of the instance name 'penguin', we obtain a high frequency corresponding to this class-instance pair, indicating that 'penguin' may belong to the class 'animal'. Likewise, we query this database for the mentions identified earlier and find the score for it to be an animal. In particular, we query for records in which the lemma of instance is the head word of the mention and lemma of the class is 'animal'. When the score obtained is greater than a certain threshold, we conclude that it is an animal. It is interesting that with this approach, the pronouns such as he, she, him, her etc. also had a high frequency for the animal class, indicating that these words are relevant for animals. Note that this approach is quite general and can be applied to any class of objects such as furniture, electronics etc.

\footnotetext{
5 This coreference resolution system is deterministic and does not provide the probabilities or strengths among mentions that is essential for our method. This prevents us from using this method from the start.
} 


\section{Implementation Details}

The pre-processing on the vision side is as in Venkitasubramanian et al. [42]. Shot cut detection and keyframe extraction are performed using [18]. Subsequently, visual features are extracted using the powerful Convolutional Neural Networks (CNN) [19]. In particular, we use the CNN-M-128 architecture of [5], which is trained on 1,000 object categories from ImageNet [8] with roughly $1.2 \mathrm{M}$ training images. With this representation, the activities of the penultimate layer (7th fully connected layer) are used as features. This model yields 128 features.

The pre-processing on the text side comprises three steps: 1) identifying animal names; 2) detecting mentions; and 3) detecting the mentions pertinent to animals. First, to identify animal names such as lion and zebra, we use the entity recognition step as described in [11]. Basically, we stem the words in the subtitles and compare them against a list of animals from WordNet [28] to obtain the animals mentioned in the subtitles. Second, to detect the mentions from the subtitles, we use the coreference resolution system of Durrett and Klein [9]. The third and final step of the text preprocessing is to refine these mentions to retain only those that are pertinent to animals. This is described in Section 5.

To build the ground truth animal mentions for the evaluation, we manually refined the list of mentions predicted by the coreference resolution system of Durrett and Klein [9] and tagged them with the correct animal from the list of animals in WordNet [28].

For building the graphs and performing the inference, we used the implementation of [39].

\section{Results}

The dataset used in our experiments is that of [42]. This is a wildlife documentary named 'Great Wildlife Moments' ${ }^{6}$ with subtitles from the BBC. This is an interlaced video with a duration of 108 minutes at a frame rate of 25 frames per second, and the frame resolution is $720 \times 576$ pixels. The video consists of 28 chapters and all the chapters except the ones containing just one animal are evaluated. This leaves us with chapters 14 to 28. Applying shot cut detection [18] on these chapters, we obtained 602 key frames. Of these, 302 frames had no animal. The remaining 300 contained 365 animals in total. We run our algorithm on all the 602 frames. Figure 5 shows some sample images from our dataset. There are various challenges due to the nature of animals (flexible bodies, self-occluding, displaying large variation in pose) and due to the nature of the video set up (natural habitat accounts for camouflage and occlusion by environmental elements).

On the textual side, the subtitles contain 7,304 words in 545 sentences. There are 206 annotated mentions pertaining to animals, of which 89 are pronouns and 41 names of animals (including repetitions). There are 19 unique animal names in total. The graph built using the textual and visual nodes over the entire dataset has 826 nodes and 3585 edges.

\footnotetext{
${ }^{6}$ https://en.wikipedia.org/wiki/Great_Wildlife_Moments
} 

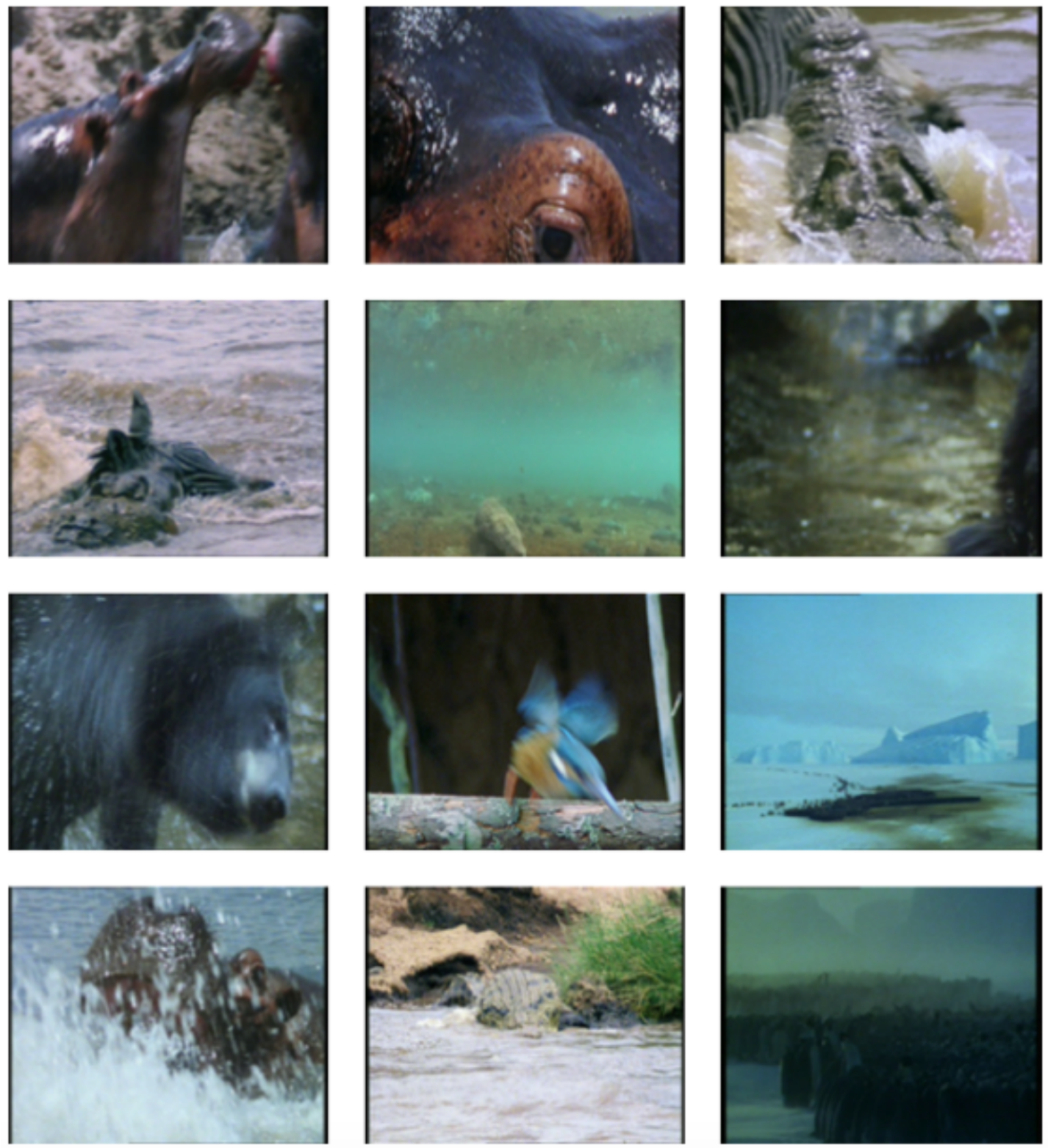

Fig. 5 Challenges from the vision side: (i) Random poses where key distinguishing features of the animal are absent, (ii) Multiple species in same image, (iii) Blurry images, (iv) The animal to be recognized is blurred out, (v) The animal to be recognized is too far from the camera, (vi) Self occlusion, (vii) Poor illumination, (viii) Occlusion due to environment/ context and camouflage. All pictures depicted above have one or more of these issues.

The evaluation covers three aspects: 1) evaluation of mention detection; 2) evaluation of entity linking; and 3) evaluation of the animal labeling.

\subsection{Detecting Relevant Mentions}

Table 1 shows the results of the mention identification using the animacy feature of [24]. The performance is quite good in terms of both precision and recall. Recall that we also used WordNet's [28] animal list to ensure that noun phrases containing 


\begin{tabular}{|c|c|c|}
\hline Precision & Recall & F1 \\
\hline 91.05 & 84.8 & 87.81 \\
\hline
\end{tabular}

Table 1 Results of the Mention Identification using the 'animacy' feature described in Section 5

\begin{tabular}{|l|c|c|c|}
\hline Threshold & Precision & Recall & F1 \\
\hline 1 & 39.55 & 95.58 & 55.95 \\
12 & 43.41 & 92.15 & 59.02 \\
100 & 56.25 & 88.23 & 68.7 \\
150 & 60.62 & 85.29 & 70.87 \\
190 & 63.46 & 84.31 & 72.42 \\
195 & 63.56 & 83.82 & 72.30 \\
$\mathbf{2 0 0}$ & $\mathbf{6 3 . 8 0}$ & $\mathbf{8 3 . 8 2}$ & $\mathbf{7 2 . 4 5}$ \\
250 & 60.85 & 70.09 & 65.14 \\
300 & 61.13 & 68.62 & 64.66 \\
\hline
\end{tabular}

Table 2 Results of the mention detection using the Hypernym Database WebIsADb [40]

animal names such as the mink, the penguins, a young white bear were not classified as INANIMATE. Most of the errors were due to the absence of a perfect match between the mentions predicted by the two coreference resolution systems ([9] and [24]), often due to different segmentation of the candidate mentions. For instance, the total number of mentions predicted by the system of Durrett and Klein [9] was 560, whereas that by Lee et al. [24] was 526, including INANIMATEs and UNKNOWNs. As an example, consider the sentence below:

Each female times her return to coincide with the hatching of her chick.

Mention identified by system of Lee et al. [24]: 'Each female times her return

to coincide with the hatching of her chick'

Mentions identified by system of Durrett and Klein [9]: 'Each female times

her' and 'the hatching of her chick'

Some of the other misses were due to forms of 'it' being classified as INANIMATE when they actually referred to animals. For example, 'To get off the beach, the killer has to thrash its body'. But, it doesn't make sense to override the animacy prediction of [24] and include all forms of 'it', since 'it' is often used as a syntactic expletive (for example, $\underline{I t}$ is raining). Other misses include more general classes of animal names such as mammals, cubs etc. There were also some false positives, where an INANIMATE concept was classified as ANIMATE, based on the context. For example, It's a way offending off evil spirits. There were a few instances where a mention is indeed animate, but does not refer to animal(s). For example, The Ainu celebrate this special event with their own dance. Both The Ainu and their were correctly classified as ANIMATE, but were not relevant for our problem. Apart from these rare cases, this approach worked quite well. It is interesting to note that some of the mentions like the intruder, the homeless female, this bedraggled survivor, these males, the other youngster were correctly identified as ANIMATE.

Next, we evaluate the mention identification using the Hypernym Database WebIsADb [40]. Table 2 shows the results for different thresholds. The threshold is basically the frequency above which a pair can be considered as a hyponym-hypernym pair. It makes sense that increasing the threshold increases the precision and decreases 
the recall. It is interesting that in most cases (threshold $\sim 25$ and above) mentions containing victims, targets etc. were also classified as ANIMATE which is useful in our context. However, there is also noise, for example, rivers seem to have a high association with animals in this database, but are nevertheless irrelevant for our problem. The best results ( $\mathrm{F} 1$ of $72.5 \%$ ) obtained when the threshold is set at 200, is still $\sim 15 \%$ short of those of the earlier approach. Nevertheless, this approach has the advantage that it could be applied to any class of objects, and is not restricted to sentient beings.

\subsection{Entity Linking in Text}

To evaluate the entity linking on text, we consider three scenarios: 1) using gold mentions; 2) using the mentions detected using the 'animacy' feature in Section 5.1 and 3) using the mentions detected using the hypernym database in Section 5.2. In either case, we experiment with two methods of initializing the vision nodes: 1) using binarized CNN features pre-trained on ImageNet as in [42]; and 2) using the output of [42].

Init is the baseline obtained by using the back-pointer probabilities from coreference resolution system of Durrett and Klein [9] $]^{7}$. This system uses only the text, (that is the subtitles in our context) and does not incorporate any visual inputs to resolve coreferences. LBP refers to Loopy Belief Propagation. Gibbs and VIT refer to Gibbs sampling and Viterbi Approximation respectively. For evaluating the textual task, we use the standard metrics [34] for coreference resolution - MUC [43], B ${ }^{3}$ [3], and $\mathrm{CEAF}_{e}$ [27], as well as their average, the CoNLL metric, all computed from the reference implementation of the CoNLL scorer [33] (see appendix for an overview of these metrics). We evaluate our methods only on the mentions that are ambiguous, that is, we leave out the animal names such as zebra, crocodile etc.

Table 3 shows the results of the entity linking on all the gold mentions (nominal and pronominal). Gold mentions are the ground-truth (manually annotated) mentions pertinent to animals. In Table 3, comparing the two methods of initializing the vision nodes, we see that using the output of [42] gives a significantly better performance. This is because these outputs give a better indication of the presence or absence of an animal in a key frame, as shown in [42]. Thus, better vision probabilities lead to better performance on the language task.

We performed a qualitative analysis to understand how the vision helps to better disambiguate the text. We found that mentions such as the male, the female and the bird are used throughout the subtitles, but refer to different animals at different points in time. For example, the bird refers to a penguin at a certain point, and then refers to a Japanese crane, a few sentences later. The use of associated video key frames helps disambiguate ambiguous mentions such as these.

Comparing the outputs of the Init system with ours, we found that some of the mistakes in the Init system are due to difficulties arising out of spoken text. In the sub-

\footnotetext{
7 Here, we look at the initial set of node potentials (obtained using the back-pointer probabilities from the coreference resolver of Durrett and Klein [9]) and assign each mention to the name with largest probability.
} 
titles, there are no paragraph breaks. As a result, the end of a topic and the beginning of the next are not clear. For example, consider the subtitle snippet below.

The splash tetra must have the most labour-intensive childcare of any fish.

But his eggs are safer from predators on leaves rather than in the river.

After two days of hard splashing, the fry emerge.

Within minutes, this nervous herd will fragment into hundreds of individual

families, as each stallion attempts to shepherd his mares and foals across.

In this example, the first three sentences are about the splash tetra, while the last is about the zebra. In spoken text such as subtitles, there are no markers to indicate the change of topic.

Sentence structures may also be different in spoken text, which makes it difficult for most coreference systems trained on well-written documents (e.g., news articles), such as that of Durrett and Klein [9]. For example,

In the rivers and lakes of Africa lives an animal which has a reputation for being the most unpredictable and dangerous of all.

Even crocodiles are wary.

The hippopotamus.

Supported by the water, they $y_{1}$ use less energy than they would on land. Moving requires only a gentle push. They save energy in other ways too.

In the example above, both they $y_{1}$ and they $y_{2}$ refer to the hippopotamus; the system of Durrett and Klein [9] maps them to crocodile while our approach identifies them correctly. The use of the visuals, together with the joint learning clearly overcomes these issues.

Second, comparing the different algorithms LBP, Gibbs and VIT, their performances are somewhat similar, although LBP is the best when using the output of [42]. In any case, using any algorithm, any mode of vision initialization, the methods outperform the initialization (Init) based on the coreference resolution in text [9] by a significant margin. LBP with an average of $83.2 \%$, initialized with [42] has a gain of over $4 \%$ compared to the baseline at $79.2 \% 8$.

Table 4 shows the results of the entity linking on all the pronominal gold mentions. The findings here are consistent with those of Table 3. Comparing these results with Table 3, note that the Average $F_{1}$ is better than those of Table 3. In general, resolution of pronouns such as he, she, their etc. is easier than resolution of mentions such as targets for the crocodiles, the victim or this bedraggled survivor.

Table 5 shows the results of the entity linking on all the mentions (nominal and pronominal) detected using the animacy feature. Again, we have the same trend as Tables 3 and 4 . There is a significant increase in average $F_{1}$ compared to the baseline that uses the text-only coreference resolution of Durrett and Klein [9]. Also, better initialization of vision nodes leads to better performance on text. Note that these results are in general lower than those reported in Tables 3 and 4 . The reason is that here we have used the mentions detected automatically using Section 5.1, instead

\footnotetext{
8 We tested the statistical significance of the results using a mention-level paired t-test and found that the LBP method was significantly better than Init $(p<0.01)$
} 


\begin{tabular}{|c|c|c|c|c|c|c|}
\hline Method & $\mathrm{B}^{3}$ & \multicolumn{3}{|c|}{ MUC } & $\mathrm{CEAF}_{e}$ & Avg \\
\hline & F1 & $\mathrm{P}$ & $\mathrm{R}$ & F1 & $\mathrm{F} 1$ & F1 \\
\hline Init & 94.3 & 69.91 & 69.23 & 69.57 & 73.77 & 79.213 \\
\hline \multicolumn{7}{|c|}{ Initialization of Vision nodes using ImageNet [8] as in [42] } \\
\hline LBP & 92.74 & 73.55 & 73.43 & 73.49 & 75.00 & 80.41 \\
\hline Gibbs & 94.3 & 76.18 & 73.64 & 74.89 & 74.8 & 81.33 \\
\hline VIT & 94.3 & 75.48 & 72.27 & 73.84 & 76.43 & 81.52 \\
\hline \multicolumn{7}{|c|}{ Initialization of Vision nodes using the output of [42] } \\
\hline LBP & 94.81 & 77.03 & 76.8 & 76.91 & 78.01 & 83.24 \\
\hline Gibbs & 94.81 & 77.10 & 75.32 & 76.20 & 76.33 & 82.45 \\
\hline VIT & 94.81 & 77.49 & 74.26 & 75.84 & 78.01 & 82.89 \\
\hline
\end{tabular}

Table 3 Results of the entity linking task using all gold mentions - nominal and pronominal

\begin{tabular}{|l|c|c|c|c|c|c|}
\hline Method & $\mathrm{B}^{3}$ & \multicolumn{3}{|c|}{ MUC } & CEAF $_{e}$ & Avg \\
\hline & $\mathrm{F} 1$ & $\mathrm{P}$ & $\mathrm{R}$ & $\mathrm{F} 1$ & $\mathrm{~F} 1$ & $\mathrm{~F} 1$ \\
\hline Init & 95.33 & 85.52 & 77.61 & 81.37 & 74.49 & 83.73 \\
\hline \multicolumn{7}{|c|}{ Initialization of Vision nodes using ImageNet [8] as in [42] } \\
\hline LBP & 95.33 & 85.52 & 78.18 & 81.69 & 75.59 & 84.20 \\
Gibbs/VIT & 95.33 & 86.11 & 79.46 & 82.65 & 77.41 & $\mathbf{8 5 . 1 3}$ \\
\hline \multicolumn{7}{|c|}{ Initialization of Vision odes using the output of [42] } \\
\hline LBP & 95.33 & 85.72 & 78.8 & 82.11 & 76.55 & 84.66 \\
Gibbs/VIT & 95.33 & 86.11 & 79.46 & 82.65 & 77.41 & $\mathbf{8 5 . 1 3}$ \\
\hline
\end{tabular}

Table 4 Results of the entity linking task using gold pronouns

of using the gold mentions. So, the errors due to mention identification are also included. Nevertheless, there are significant improvements over the baseline, with $4 \%$ improvement in $F_{1}$ measure, while using LBP with initialization based on [42]. ${ }^{9}$

Table 6 shows the results of the entity linking on all the mentions (nominal and pronominal) detected using the hypernym database, with the threshold set to 200 based on the results in Table 2. As before, we note the significant increase in average $F_{1}$ compared to the baseline that uses the text-only coreference resolution of Durrett and Klein [9]. These results are also lower than those based on the animacy based approach (reported in Table 5) by about $14 \%$. Recall that the $F_{1}$ of the hypernym based mention detection is $\sim 15 \%$ lower than that of the animacy based approach.

Figure 6 shows some sample entity labels generated by our system using gold mentions and LBP. Note that the system correctly resolves the ambiguous nominals such as a big land animal, some foals etc. Some of the errors in our system occur when there were multiple animals in a frame, and neither the back-pointer probabilities [9] in text nor the vision probabilities from [42] gave a good estimate of the probability of the animal name given the frame/mention to start with. One such example is the misclassification of many to crocodiles when they actually referred to zebras (Figure 6). The last text excerpt in this figure is really interesting. All the pronouns in this example actually refer to mink, but just looking at the text leads us to believe that they refer to the kingfisher. It is impossible to resolve these mentions correctly without the vision.

\footnotetext{
9 The global inferencing over text and vision for the entire video was quite fast. On an Intel Xeon CPU E5-2687W processor with $3.10 \mathrm{GHz}$, the LBP took 0.65997 seconds, while VIT and Gibbs took 0.76278 and 0.67559 seconds respectively.
} 


\begin{tabular}{|c|c|}
\hline $\begin{array}{l}\text { In the rivers and lakes of Africa lives an animal } \\
\text { which has a reputation for being the most } \\
\text { unpredictable and dangerous of all }{ }_{1} \text {. } \\
\text { Even crocodiles are wary. } \\
\text { The hippopotamus. } \\
\text { Supported by the water, they } y_{2} \text { use less energy } \\
\text { than they would on land. }\end{array}$ & $\begin{array}{l}\text { 1. an animal which has a reputation for } \\
\text { being the most unpredictable and } \\
\text { dangerous of all } \\
\text { GT: hippopotamus } \\
\text { Predicted: hippopotamus } \\
\text { 2. they } \\
\text { GT: hippopotamus } \\
\text { Predicted: hippopotamus }\end{array}$ \\
\hline $\begin{array}{l}\text { There's more than one young bear around, and } \\
\text { some lessons have to be learned by way of a } \\
\text { mistake. } \\
\text { But for this youngster }{ }_{1} \text {, it's worth checking to } \\
\text { see if the other youngster }{ }_{2} \text { knows something } \text { he }_{3} \\
\text { doesn't. } \\
\text { But no. A truth is confirmed. } \\
\text { In the water, a big land animal }{ }_{4} \text { is no match for } \\
\text { quick slippery fishs. }\end{array}$ & $\begin{array}{l}\text { 1. this youngster } \\
\text { GT: bear } \\
\text { Predicted: bear } \\
\text { 2. the other youngster } \\
\text { GT: bear } \\
\text { Predicted: bear } \\
\text { 3. he } \\
\text { GT: bear } \\
\text { Predicted: bear } \\
\text { 4. a big land animal } \\
\text { GT: bear } \\
\text { Predicted: bear } \\
\text { 5. quick slippery fish } \\
\text { GT: salmon } \\
\text { Predicted: salmon }\end{array}$ \\
\hline $\begin{array}{l}\text { Some foals } s_{1} \text { are strong enough to defend } \\
\text { themselves } s_{2} \text { in spite of the crocodiles' } \\
\text { determination, and many } \text { succeed in repelling }_{3} \text { succer } \\
\text { their } \text { attackers }_{5} \text {. }\end{array}$ & $\begin{array}{l}\text { 1. Some foals } \\
\text { GT: zebra } \\
\text { Predicted: zebra } \\
\text { 2. themselves } \\
\text { GT: zebra } \\
\text { Predicted: zebra } \\
\text { 3. many } \\
\text { GT: zebra } \\
\text { Predicted: crocodile } \\
\text { 4. their } \\
\text { GT: zebra } \\
\text { Predicted: zebra } \\
\text { 5. their attackers } \\
\text { GT: crocodile } \\
\text { Predicted: crocodile }\end{array}$ \\
\hline $\begin{array}{l}\text { But one kingfisher got lucky. } \\
\text { She }{ }_{1} \text { spotted me. We were so absorbed in the } \\
\text { fight that she's } s_{2} \text { as surprised to see me as I was } \\
\text { to see her }{ }_{3} \text {. }\end{array}$ & $\begin{array}{l}\text { 1. She } \\
\text { GT: mink } \\
\text { Predicted: mink } \\
\text { 2. She } \\
\text { GT: mink } \\
\text { Predicted: mink } \\
\text { 3. her } \\
\text { GT: mink } \\
\text { Predicted: mink }\end{array}$ \\
\hline
\end{tabular}

Fig. 6 Some sample outputs from our system using gold mentions and LBP. The left column shows the subtitle text. Bolded mentions contain an animal name, while the underlined mentions are the ones to be resolved. The right column shows the outputs. 'GT' indicates ground truth, 'Predicted' indicates the predictions of the system. 


\begin{tabular}{|c|c|c|c|c|c|c|c|c|c|c|}
\hline Method & \multicolumn{3}{|c|}{$\mathrm{B}^{3}$} & \multicolumn{3}{|c|}{ MUC } & \multicolumn{3}{|c|}{$\mathrm{CEAF}_{e}$} & Avg \\
\hline & $\mathrm{P}$ & $\mathrm{R}$ & F1 & $\mathrm{P}$ & $\mathrm{R}$ & F1 & $\mathrm{P}$ & $\mathrm{R}$ & F1 & F1 \\
\hline Init & 78.23 & 84.83 & 81.4 & 50.98 & 57.46 & 54.03 & 57.39 & 52.61 & 54.9 & 63.44 \\
\hline \multicolumn{11}{|c|}{ Initialization of Vision nodes using ImageNet [8] as in [42] } \\
\hline LBP & 77.2 & 83.7 & 80.32 & 52.69 & 61.08 & 56.58 & 57.91 & 53.09 & 55.39 & 64.10 \\
\hline Gibbs & 79.27 & 85.95 & 82.47 & 57.72 & 65.82 & 61.5 & 61.61 & 56.47 & 58.93 & 67.63 \\
\hline VIT & 78.75 & 85.39 & 81.94 & 56 & 62.33 & 58.99 & 60.37 & 55.34 & 57.74 & 66.22 \\
\hline \multicolumn{11}{|c|}{ Initialization of Vision nodes using the output of [42] } \\
\hline LBP & 79.27 & 85.95 & 82.47 & 57.92 & 64.24 & 60.92 & 61.94 & 56.78 & 59.25 & 67.55 \\
\hline Gibbs & 79.27 & 85.95 & 82.47 & 58.36 & 64.33 & 61.2 & 61.17 & 56.07 & 58.51 & 67.39 \\
\hline VIT & 79.27 & 85.95 & 82.47 & 56.17 & 63.75 & 59.72 & 59.98 & 54.98 & 57.38 & 66.52 \\
\hline
\end{tabular}

Table 5 Results of the Entity Linking on all mentions (nominal and pronominal) detected using the animacy feature of [24]

\begin{tabular}{|l|c|c|c|c|c|c|c|c|c|c|}
\hline Method & \multicolumn{3}{c|}{$\mathrm{B}^{3}$} & \multicolumn{3}{c|}{ MUC } & \multicolumn{3}{c|}{ CEAF $_{e}$} & Avg \\
\hline & $\mathrm{P}$ & $\mathrm{R}$ & $\mathrm{F} 1$ & $\mathrm{P}$ & $\mathrm{R}$ & $\mathrm{F} 1$ & $\mathrm{P}$ & $\mathrm{R}$ & $\mathrm{F} 1$ & $\mathrm{~F} 1$ \\
\hline Init & 78.75 & 59.37 & 67.70 & 56.4 & 33.16 & 41.77 & 44.99 & 44.99 & 44.99 & 51.49 \\
\hline \multicolumn{10}{|c|}{ Initialization of Vision nodes using ImageNet [8] as in [42] } \\
\hline LBP & 78.75 & 59.37 & 67.70 & 60.58 & 34.93 & 44.31 & 48.40 & 48.40 & 48.40 & 53.47 \\
Gibbs & 78.75 & 59.37 & 67.70 & 59.52 & 34.28 & 43.51 & 47.06 & 47.06 & 47.06 & 52.76 \\
VIT & 78.75 & 59.37 & 67.70 & 56.62 & 33.61 & 42.19 & 45.80 & 45.80 & 45.80 & 51.90 \\
\hline \multicolumn{10}{|c|}{ Initialization of Vision nodes using the output of [42] } \\
\hline LBP & 79.27 & 59.76 & 68.15 & 62.99 & 35.25 & 45.2 & 48.22 & 48.22 & 48.22 & $\mathbf{5 3 . 8 6}$ \\
Gibbs & 78.75 & 59.37 & 67.7 & 61.21 & 35.23 & 44.72 & 48.60 & 48.60 & 48.60 & 53.67 \\
VIT & 79.27 & 59.76 & 68.15 & 58.00 & 34.66 & 43.39 & 47.49 & 47.49 & 47.49 & 53.01 \\
\hline
\end{tabular}

Table 6 Results of the Entity Linking on all mentions (nominal and pronominal) detected using the Hypernym Database WebIsADb [40]

\subsection{Animal Labeling on Vision}

As with the entity liking on text, we experiment with three scenarios: 1) Gold mentions, 2) mentions identified using the animacy feature and 3) mentions identified using the hypernym database. Table 7 shows the results of the animal labeling on vision for both the scenarios. The baseline (Init) is the initialization obtained using the output probabilities of Venkitasubramanian et al. [42]. Recall that the approach of [42] learns classifiers from ImageNet [8] and iteratively adapts them to the target dataset using textual cues from subtitles, particularly whether or not a certain animal name is mentioned in the subtitles corresponding to each frame in the target dataset. Their system outputs a set of probabilities $p\left(n_{k} \mid f_{j}\right)$ that any frame $f_{j}$ contains animal $n_{k}$. The baseline (Init) that we used assumes that an animal $n_{k}$ is present in frame $f_{j}$ if the corresponding probability $p\left(n_{k} \mid f_{j}\right)>0.5$.

While in the setting of [42] there was ambiguity due to the lack of reliable connections between the animal and name pairs across text and vision, here, we deal with even more ambiguity, since we have mentions such as 'the female'. Despite that, we have significantly better performance with both gold and detected mentions. This is because improving the coreference resolution leads to more confident animal imagename pairs. 
The performance of the three methods (LBP, VIT and Gibbs) are comparable, although LBP gives the best performance with a gain of close to $4 \%$ in F1 compared to the Init baseline ${ }^{10}$.

Further, when comparing the performance of the system using gold mentions with that using detected mentions, we note that the former is clearly better. This makes sense because false positive mentions (e.g. 'evil spirits' and 'Ainu' were classified as ANIMATE) will still be mapped to animal names on text and their probabilities will impact the rest of the graph. Better text leads to better results on the vision side.

Another interesting aspect of the evaluation regards the performance of the algorithm when more than one name is associated with a frame. In our dataset, there are 65 frames with more than one animal shown, and 153 frames with more than one possible name assigned as weak labels (using the nearby subtitles). Using gold mentions with the LBP algorithm, we obtained a precision of $82.46 \%$ and recall of $83.93 \%$ (yielding an $\mathrm{F} 1$ of $83.19 \%$ ) in the first case, and a precision of $59.14 \%$ and recall of $91.67 \%$ (yielding an $\mathrm{F} 1$ of $71.90 \%$ ) in the second case. The latter case is harder, making the F1 slightly lower than that on the entire dataset (73.42\%). While these cases are certainly challenging, even identifying frames with just one animal or none is not straightforward, since it is not known in advance whether or not there is an animal in the frame.

Yet another aspect of the evaluation is the impact of joint modelling. To measure this, we experiment with the system of Venkitasubramanian et al. [42] using the model proposed here to resolve entity mentions. Recall that the system of Venkitasubramanian et al. [42] uses a combination of coreference resolvers to resolve pronouns. The outputs of all the coreference systems were fed into an EM algorithm, which then used these as evidence to connect an animal image-name pair. We ran this system using our entity labels, instead of the combination of coreference resolvers. In this case, we obtained an F1 of $65.59 \%$ (Second row in table 7) for the animal labeling task. These results are lower compared to those obtained in [42], which was based on multiple coreference resolvers. This is because, in our setup, we consider more complex mentions including nominals which are ambiguous, instead of just using pronouns (which are easier to resolve compared to the ambiguous nominals we have in our dataset) and animal names. Some of the errors we obtained in the entity linking propagated through the system of [42], resulting in the lower performance. Comparing the approach that we propose in this paper with the method of [42], we see a significant increase in the performance of the animal labeling, which is largely attributed to the joint modelling. The improvement on the vision side is not just because of the better entity labels, but also due to the joint modelling.

Figure 7 shows some outputs of our system with LBP. Our system performs quite well despite various challenges such as blurry subject, random poses etc. The system is capable of identifying multiple species in the same frame, as well as detecting frames that do not contain any animal.

\footnotetext{
10 We tested the statistical significance of the results using a frame-level paired t-test and found that the LBP method was significantly better than Init both in terms of precision $(p<0.001)$ and recall $(p=0.0093)$
} 


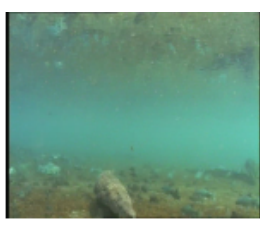

GT: Crocodile

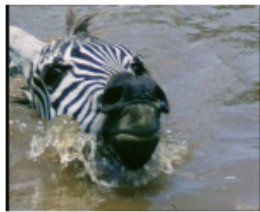

GT: Zebra

Predicted: Zebra, Crocodile

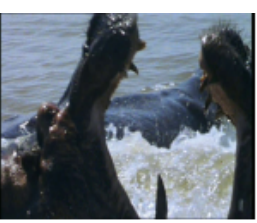

GT: Hippopotamus Predicted: Hippopotamus

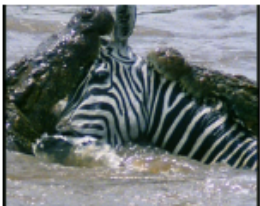

GT: Zebra, Crocodile

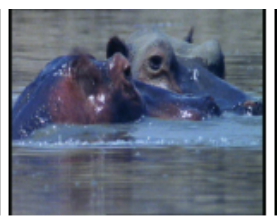

GT: Hippopotamus

Predicted: Hippopotamus

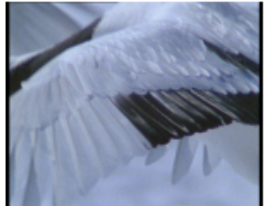

GT: Crane

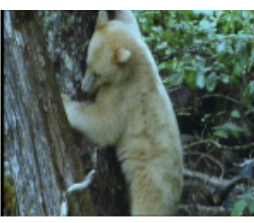

GT: Bear

Predicted:Bea

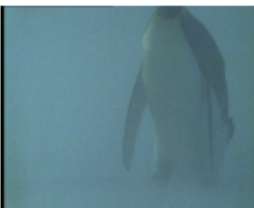

GT: Penguin

Predicted: Penguin

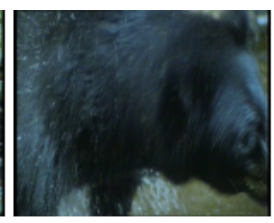

GT: Bear

Predicted: Bear

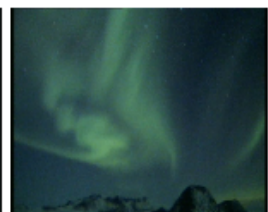

GT: -

Predicted: -

Fig. 7 Some sample outputs from our system using LBP. 'GT' indicates ground truth, 'Predicted' indicates the predictions of the system.

\begin{tabular}{|l|c|c|c|}
\hline Method & Precision & Recall & F1 \\
\hline Init & 57.27 & 88.73 & 69.61 \\
Approach of [42] using our entity labels & 53.22 & 85.45 & 65.59 \\
\hline \multicolumn{4}{|c|}{ Using gold mentions } \\
\hline LBP & 65.99 & 82.74 & $\mathbf{7 3 . 4 2}$ \\
Gibbs & 61.84 & 88.83 & 72.92 \\
VIT & 62.83 & 85.79 & 72.53 \\
\hline \multicolumn{4}{|c|}{ Using mentions detected based on the animacy feature } \\
\hline LBP & 58.18 & 89.72 & $\mathbf{7 0 . 5 9}$ \\
Gibbs & 58.23 & 89.25 & 70.48 \\
VIT & 57.52 & 91.12 & 70.52 \\
\hline \multicolumn{4}{|c|}{ Using mentions detected based on the hypernym database } \\
\hline LBP & 59.30 & 86.45 & $\mathbf{7 0 . 3 4}$ \\
Gibbs & 59.42 & 85.51 & 70.12 \\
VIT & 57.31 & 89.72 & 69.95 \\
\hline
\end{tabular}

Table 7 Results of the animal labeling task on the visual data using gold mentions and mentions detected automatically

\section{Conclusions}

This paper shows that the joint modelling of entity linking tasks in vision and language results in better performance in both modalities. The framework proposed incorporates beliefs from state-of-the-art methods independently from text and vision, and performs global inference over the whole video using a structured predictor, to perform entity linking in language and vision. We have shown that the performance of the entity linking has improved through the use of visual cues while that of the animal labeling has improved through the use of better textual coreference resolution. Furthermore, we have demonstrated the use of our method for textual mentions that cannot be resolved using text-only methods. While the framework has been validated on a wildlife documentary here, the methods proposed are quite generic and can be 
applied to various other scenarios involving language and vision, such as aligning people's names with faces or furniture and other objects.

In the future, we wish to apply these methods to other datasets involving a wide variety of subjects. Further, we would like to extract the interesting regions in the picture which can contribute to a better performance. The approaches described advance us towards automatic multimedia indexing.

\section{References}

1. Afkham, H.M., Targhi, A.T., Eklundh, J.O., Pronobis, A.: Joint visual vocabulary for animal classification. In: 19th International Conference on Pattern Recognition, pp. 1-4. IEEE (2008)

2. Alfonseca, E., Manandhar, S.: An unsupervised method for general named entity recognition and automated concept discovery. In: Proceedings of the 1st international conference on general WordNet, Mysore, India, pp. 34-43 (2002)

3. Bagga, A., Baldwin, B.: Algorithms for scoring coreference chains. In: The first international conference on language resources and evaluation workshop on linguistics coreference, vol. 1, pp. 563-566. Citeseer (1998)

4. Berg, T.L., Forsyth, D.A.: Animals on the web. In: Computer Vision and Pattern Recognition, 2006 IEEE Computer Society Conference on, vol. 2, pp. 1463-1470. IEEE (2006)

5. Chatfield, K., Simonyan, K., Vedaldi, A., Zisserman, A.: Return of the devil in the details: Delving deep into convolutional nets. arXiv preprint arXiv:1405.3531 (2014)

6. Coates-Stephens, S.: The analysis and acquisition of proper names for the understanding of free text. Computers and the Humanities 26(5-6), 441-456 (1992)

7. Coughlan, J.M., Ferreira, S.J.: Finding deformable shapes using loopy belief propagation. In: European Conference on Computer Vision, pp. 453-468. Springer (2002)

8. Deng, J., Dong, W., Socher, R., Li, L.J., Li, K., Fei-Fei, L.: Imagenet: A large-scale hierarchical image database. In: Computer Vision and Pattern Recognition, 2009. CVPR 2009. IEEE Conference on, pp. 248-255. IEEE (2009)

9. Durrett, G., Klein, D.: Easy victories and uphill battles in coreference resolution. In: Proceedings of the Conference on Empirical Methods in Natural Language Processing. Association for Computational Linguistics, Seattle, Washington (2013)

10. Durrett, G., Klein, D.: A joint model for entity analysis: Coreference, typing, and linking. In: Transactions of the Association for Computational Linguistics (2014)

11. Dusart, T., Nurani Venkitasubramanian, A., Moens, M.F.: Cross-modal alignment for wildlife recognition. In: Proceedings of the 2nd ACM International Workshop on Multimedia Analysis for Ecological Data, pp. 9-14. ACM (2013)

12. Everingham, M., Van Gool, L., Williams, C.K.I., Winn, J., Zisserman, A.: The PASCAL Visual Object Classes Challenge 2012 (VOC2012) Results. http://www.pascalnetwork.org/challenges/VOC/voc2012/workshop/index.html

13. Fang, H., Gupta, S., Iandola, F., Srivastava, R., Deng, L., Dollár, P., Gao, J., He, X., Mitchell, M., Platt, J., et al.: From captions to visual concepts and back. arXiv preprint arXiv:1411.4952 (2014)

14. Gomez, A., Salazar, A.: Towards automatic wild animal monitoring: Identification of animal species in camera-trap images using very deep convolutional neural networks. arXiv preprint arXiv:1603.06169 (2016)

15. Guadarrama, S., Krishnamoorthy, N., Malkarnenkar, G., Venugopalan, S., Mooney, R., Darrell, T., Saenko, K.: Youtube2text: Recognizing and describing arbitrary activities using semantic hierarchies and zero-shot recognition. In: Computer Vision (ICCV), 2013 IEEE International Conference on, pp. 2712-2719. IEEE (2013)

16. Guillaumin, M., Mensink, T., Verbeek, J., Schmid, C.: Automatic face naming with caption-based supervision. In: Computer Vision and Pattern Recognition, 2008. CVPR 2008. IEEE Conference on, pp. 1-8. IEEE (2008)

17. Hariharan, B., Girshick, R.: Low-shot visual object recognition. arXiv preprint arXiv:1606.02819 (2016)

18. Hellier, P., Demoulin, V., Oisel, L., Pérez, P.: A contrario shot detection. In: 2012 19th IEEE International Conference on Image Processing, pp. 3085-3088. IEEE (2012) 
19. Joly, A., Goëau, H., Glotin, H., Spampinato, C., Bonnet, P., Vellinga, W.P., Planqué, R., Rauber, A., Palazzo, S., Fisher, B., et al.: Lifeclef 2015: multimedia life species identification challenges. In: International Conference of the Cross-Language Evaluation Forum for European Languages, pp. 462-483. Springer (2015)

20. Karpathy, A., Fei-Fei, L.: Deep visual-semantic alignments for generating image descriptions. arXiv preprint arXiv:1412.2306 (2014)

21. Kazemzadeh, S., Ordonez, V., Matten, M., Berg, T.L.: Referit game: Referring to objects in photographs of natural scenes. In: EMNLP (2014)

22. Khosla, A., Jayadevaprakash, N., Yao, B., Li, F.f.: L.: Novel dataset for fine-grained image categorization. In: First Workshop on Fine-Grained Visual Categorization, CVPR (2011. Citeseer (2011)

23. Kong, C., Lin, D., Bansal, M., Urtasun, R., Fidler, S.: What are you talking about? text-to-image coreference. In: 2014 IEEE Conference on Computer Vision and Pattern Recognition, pp. 35583565. IEEE (2014)

24. Lee, H., Chang, A., Peirsman, Y., Chambers, N., Surdeanu, M., Jurafsky, D.: Deterministic coreference resolution based on entity-centric, precision-ranked rules. Computational Linguistics 39(4), 885-916 (2013)

25. Leser, U., Hakenberg, J.: What makes a gene name? named entity recognition in the biomedical literature. Briefings in bioinformatics 6(4), 357-369 (2005)

26. Liu, X., Li, Y., Wu, H., Zhou, M., Wei, F., Lu, Y.: Entity linking for tweets. In: ACL (1), pp. 1304 $1311(2013)$

27. Luo, X.: On coreference resolution performance metrics. In: Proceedings of the conference on Human Language Technology and Empirical Methods in Natural Language Processing, pp. 25-32. Association for Computational Linguistics (2005)

28. Miller, G.A.: WordNet: A lexical database for English. Communications of the ACM 38, 39-41 (1995)

29. Nadeau, D., Sekine, S.: A survey of named entity recognition and classification. Lingvisticae Investigationes 30(1), 3-26 (2007)

30. Pearl, J.: Probabilistic reasoning in intelligent systems: Networks of plausible inference. Morgan Kaufmann (2014)

31. Pham, P.T., Moens, M.F., Tuytelaars, T.: Cross-media alignment of names and faces. Multimedia, IEEE Transactions on 12(1), 13-27 (2010)

32. Pham, P.T., Tuytelaars, T., Moens, M.F.: Naming people in news videos with label propagation. IEEE Multimedia 18(3), 44-55 (2011)

33. Pradhan, S., Luo, X., Recasens, M., Hovy, E., Ng, V., Strube, M.: Scoring coreference partitions of predicted mentions: A reference implementation (2014)

34. Pradhan, S., Ramshaw, L., Marcus, M., Palmer, M., Weischedel, R., Xue, N.: Conll-2011 shared task: Modeling unrestricted coreference in ontonotes. In: Proceedings of the Fifteenth Conference on Computational Natural Language Learning: Shared Task, pp. 1-27. Association for Computational Linguistics (2011)

35. Ramanan, D., Forsyth, D.A., Barnard, K.: Building models of animals from video. Pattern Analysis and Machine Intelligence, IEEE Transactions on 28(8), 1319-1334 (2006)

36. Ramanathan, V., Joulin, A., Liang, P., Fei-Fei, L.: Linking people in videos with their names using coreference resolution. In: European Conference on Computer Vision, pp. 95-110. Springer (2014)

37. Roth, D., Yih, W.t.: Probabilistic reasoning for entity \& relation recognition. In: Proceedings of the 19th International Conference on Computational Linguistics-Volume 1, pp. 1-7. Association for Computational Linguistics (2002)

38. Schmid, C.: Constructing models for content-based image retrieval. In: Computer Vision and Pattern Recognition, 2001. CVPR 2001. Proceedings of the 2001 IEEE Computer Society Conference on, vol. 2, pp. II-39. IEEE (2001)

39. Schmidt, M.: UGM: Matlab code for undirected graphical models (2012)

40. Seitner, J., Bizer, C., Eckert, K., Faralli, S., Meusel, R., Paulheim, H., Ponzetto, S.: A large database of hypernymy relations extracted from the web. In: Proceedings of the 10th edition of the Language Resources and Evaluation Conference, Portoroz, Slovenia (2016)

41. Shen, W., Wang, J., Han, J.: Entity linking with a knowledge base: Issues, techniques, and solutions. IEEE Transactions on Knowledge and Data Engineering 27(2), 443-460 (2015)

42. Venkitasubramanian, A.N., Tuytelaars, T., Moens, M.F.: Wildlife recognition in nature documentaries with weak supervision from subtitles and external data. Pattern Recognition Letters, Elsevier, 2016 (2016) 
43. Vilain, M., Burger, J., Aberdeen, J., Connolly, D., Hirschman, L.: A model-theoretic coreference scoring scheme. In: Proceedings of the 6th conference on Message understanding, pp. 45-52. Association for Computational Linguistics (1995)

44. Wah, C., Branson, S., Welinder, P., Perona, P., Belongie, S.: The Caltech-UCSD birds-200-2011 dataset, Technical Report CNS-TR-2011-001, California Institute of Technology (2011)

\section{A Appendix: Metrics for evaluating the entity linking on text}

We denote a set of mentions referring to the same entity as an entity cluster. Given a set of key (groundtruth) entity clusters $K$, and a set of response (system-generated) entity clusters $R$, with each entity cluster comprising one or more mentions, each metric generates its variation of a precision and recall measure. The MUC measure is the oldest and most widely used. It focuses on the links (or pairs of mentions) in the data. The number of common links between elements in $K$ and $R$ divided by the number of links in $K$ represents the recall, whereas, precision is the number of common links between elements in $K$ and $R$ divided by the number of links in $R$. This metric prefers systems that have more mentions per cluster; a system that creates a single cluster of all the mentions will get a $100 \%$ recall without significant degradation in its precision. It ignores recall for singleton clusters, or entities with only one mention.

The $\mathrm{B}^{3}$ metric tries to addresses MUCs shortcomings, by focusing on the mentions and computes recall and precision scores for each mention. If $\mathrm{K}$ is the key entity cluster containing mention $\mathrm{m}$, and $\mathrm{R}$ is the response entity cluster containing mention $\mathrm{M}$, then recall for the mention $\mathrm{m}$ is computed as $\frac{|\mathrm{K} \cap \mathrm{R}|}{|\mathrm{K}|}$ and precision for the same is is computed as $\frac{|\mathrm{K} \cap \mathrm{R}|}{|\mathrm{R}|}$. Overall recall and precision are the average of the individual mention scores.

CEAF aligns every response cluster with at most one key cluster by finding the best one-to-one mapping between the clusters using an entity similarity metric. This is a maximum bipartite matching problem solved by the Kuhn-Munkres algorithm. This metric works at the level of the entity cluster. Depending on the similarity, there are two variations: a) entity based CEAF - CEAF $e$ and b) mention based CEAF $\mathrm{CEAF}_{m}$. Recall is the total similarity divided by the number of mentions in $K$, and precision is the total similarity divided by the number of mentions in $R$. In this work, we use $\mathrm{CEAF}_{e}$ for evaluation, similar to the state-of-the-art coreference resolution and entity linking systems $[9,24,10]$. 